\title{
Human Centric Building Control System
}

\author{
Kevin B. Martin \\ Department of Technology \\ Northern Illinois University, DeKalb, IL, 60115 USA \\ William Mills \\ Department of Technology \\ Northern Illinois University, DeKalb, IL, 60115 USA
}

\begin{abstract}
The Human Centric Building Control System is a focal point of the convergence of separated networks such as lighting, indoor air quality, security, Wi-Fi, and real time location through wireless and Power over Ethernet (PoE) communication. The differentiating factor of the developed building management system is emphasis on productivity and human factors while increasing energy efficiency. The integrated system includes color tunable light emitting diode (LED) luminaires, a lighting and a heating, ventilation, and air conditioning (HVAC) unit with variable speed drives (VSDs) and a Raspberry Pi microcomputers which collect data and are the control units in the system. The developed system monitors and adjusts indoor air quality (IAQ) and lighting conditions based on time of day and real time sensor measurements.
\end{abstract}

Keywords - Building Management System, Spectrally Enhanced Lighting, Indoor Air Quality

\section{INTRODUCTION}

The focus of the developed Human Centric Building Control (HCBC) system is an intelligent automated building management system which incorporates energy efficiency and human factors in its operation. Benefits of such a system include more efficient use of resources, improved worker productivity, automated environmental changes, and improved human experience. The development of such a system has recently made possible primarily due to the reduction in sensor technology and cost. A 2016 study from Intel concluded that basic building management systems (BMS) costs around $\$ 2.50$ to as high as $\$ 7.00$ per $\mathrm{ft}^{2}$ deployed [1]. The principal components of the developed system include a scaled HVAC unit, color tunable LED luminaires, real time personnel tracking system and Raspberry Pi microcomputers which act as data collection and control units. Although some types of advanced integrated systems are being commercialized such systems typically utilize proprietary communication protocols or do not fully take into account human effects.

A growing body of literature is demonstrating the effects a building environment has on workers. Mills et al. measured approximately a 30\% improvement in fatigue reduction, alertness, daytime sleepiness and an approximate $19 \%$ improvement in work performance utilizing $17000 \mathrm{~K}$ lamps compared to $2900 \mathrm{~K}$ lamps [2]. The effect of temperature was investigated by Tom which monitored worker productivity as function of indoor air temperature between $68^{\circ} \mathrm{F}$ to $77^{\circ} \mathrm{F}$ [3]. The study found an increase in productivity between $72^{\circ} \mathrm{F}$ and $77^{\circ} \mathrm{F}$ along with an associated decrease in committed errors. Concerning carbon dioxide levels, Satish et al. measured statistically significant decrements in decision making performance took place between 600-2,500 ppm [4]. Improvements in productivity (up to $11 \%$ increase) have been linked to increased outside air rates, dedicated delivery of fresh air to the workstation and reduced levels of pollutants [5].

\section{THEORY}

Both rods and cones contribute to visual perception in the human eye. Beginning in the 1990s, Berman identified that lighting measurements needed to be revised to include the contribution rods make towards visual acuity and overall perceived brightness [6]. Smaller pupils provide better acuity, improved depth of field and allow a less accommodative response of the eye. Current mainstream light meters only take into the contribution of cones to visual perception as they only utilize the photopic response curve to determine the measured illuminance (lux). The photopic response curve provides a correlation between the sensitivity of the cones in the human eye to wavelengths of light in the visual range. However, as rods control the opening and closing of the pupil, they directly have an effect on the visual performance under typical lighting conditions in the workplace. Therefore, in order to have a more realistic measurement of the actual visual performance of a luminaire the use of the scotopic response curve 
should be utilized. The scotopic response curve characterizes the correlation between sensitivity of rods in the visual light range. At a minimum the relative ratio of scotopic light versus photopic light or S/P ratio can used to calculate the equivalent pupil illuminance or sometimes referred to as the visually effective light (VEL). Using a given acceptable photopic illuminance, a light sources VEL can be calculated using Equation 1 for areas where all or most light is provided by general service luminaires [6].

$$
V E L=P x(s / p)^{0.78}
$$

The Illinois Department of Health IAQ standards for particulate state that PM10 should not be more than $150 \mu \mathrm{g} / \mathrm{m}^{3}$ and PM2.5 should be less than $65 \mu \mathrm{g} / \mathrm{m}^{3}$ for a 24 hour period. In order to actively monitor and compensate for changes the particulate sensor should not surpass the average. To keep the average low and monitor air quality a 12 $\mu \mathrm{g} / \mathrm{m}^{3}$ is used as a control limit and is outlined by an EPA study on ambient air quality [7]. Indoor Environmental Quality (IEQ) is a more general term which includes IAQ, but also considers lighting, acoustic conditions and thermal comfort and other Human Factor (HF) considerations [8]. Standards, guidelines or considerations for IEQ/IAQ have been issued by organizations such as ASHRAE [9-11], US Green Building Council (USGBC) [12], the European Committee for Standardization (CEN) [13-14], and the American Industrial Hygiene Association (AIHA) [15-16].

\section{EXPERIMENT AND RESULT}

The developed system is capable of handling various wireless and wired communications protocols along with a vast array of sensors. This is accomplished primarily as data is measured, transmitted, and analyzed using various Raspberry Pi (RPi) models and the use of a Samsung SmartThings hub. See Figure 1. Raspberry Pi communication is done through secure socket shell (SSH). Each of the Raspberry Pis have a RSA public and private key installed to allow for SSH without the use of a password. This was done by using a key generator and then saving the public key into the authorized keys file. SSH was then used to concatenate the public key to the other Raspberry Pis authorized keys file. To insure accurate measurements, an Osram RGBW LED A19 luminaire was placed inside a placed inside a black felt lined enclosure to remove internal reflectance and outside disturbances. In order to determine the appropriate settings for the luminaire, the current luminaire VEL is calculated through a characterization equation which uses CCT and dimness data that is acquired through the Samsung SmartThings ${ }^{\mathrm{TM}}$ hub. An Ocean Optics STSVIS spectrometer is used to measure the photopic lux and the spectral power distribution which is used to calculate the $\mathrm{S} / \mathrm{P}$ ratio of the environment (luminaire and simulated daylighting if present). The required change in VEL is then calculated based on a user defined set point. The main RPi then provides an updated set of operational parameters to the luminaire based on the required change.

The main RPi is also responsible for determining the operational setup points for the heating, ventilation, air conditioning (HVAC) unit and transferring the total occupancy of the room to the fan controller. The first step for the main control program is to get the occupancy data via secure copy (SCP). The room occupancy is monitored by a Raspberry Pi 2 positioned at the entrance door with three passive infrared sensors (PIR). Each sensor has a field of view of $120^{\circ}$ and can reach up to 7 meters. In order to make a more precise count the view angle was reduced to $30^{\circ}$ via a cover. The program for the total occupancy count determines which of the sensors is triggered first, the outer or inner in combination with the center PIR and either counts up by one or reduces the current in room count by one. The $\mathrm{CO}_{2}$, particulate, temperature, and humidity data are collected via SCP from an additional RPi. The in-room temperature, humidity, and $\mathrm{CO}_{2}$ are monitored by a COZAIR $\mathrm{CO}_{2}$ sensor. The COZAIR sensor provided acceptable precision when benchmarked against a 3M EVM-7. A Grove dust particulate sensor, which is designed to detect particles greater than $1 \mu \mathrm{m}$, is also connected to the same RPi. Once entering a control loop based on season, the $\mathrm{CO}_{2}$ is checked against the $1000 \mathrm{ppm}$ set point followed by the particulate level to insure the current level is below the 12 $\mu \mathrm{g} / \mathrm{m}^{3}$ set point. Based upon time of day and total occupancy, temperature and humidity set points are sent to separate RPis which communicate to HVAC unit and manage the outdoor air intake and recirculation fans. Communication with the HVAC was accomplished through pulse code modulation (PCM). The $38 \mathrm{KHz}$ carrier frequency was decoded using an IR decoder and an infrared shield on a Raspberry Pi 2. A profile for the unit was created with a default startup default configuration. To allow for short changes in occupancy and reduce equipment cycling the HVAC unit is adjusted every 5 minutes unless safety flags are triggered. If $\mathrm{CO}_{2}$ or particulate safety levels are superseded, flags are posted which trip the main controller to power on the HVAC unit with maximum fan speed and depending on which parameter is over limit the recirculation fan and possibly the outdoor fan are set to the maximum flow setting. During normal operation, the relative amounts of recirculating and outdoor air are controlled by monitoring the $\mathrm{CO}_{2}$ produced at $20 \mathrm{CFM}$ per person. $5 \mathrm{~V}$ stepper motors were mounted to separate 
variable speed controllers for the two fans to allow for independent control. To insure proper operation of the HVAC unit, the treated air temperature and humidity are measured by an AM2315 sensor.

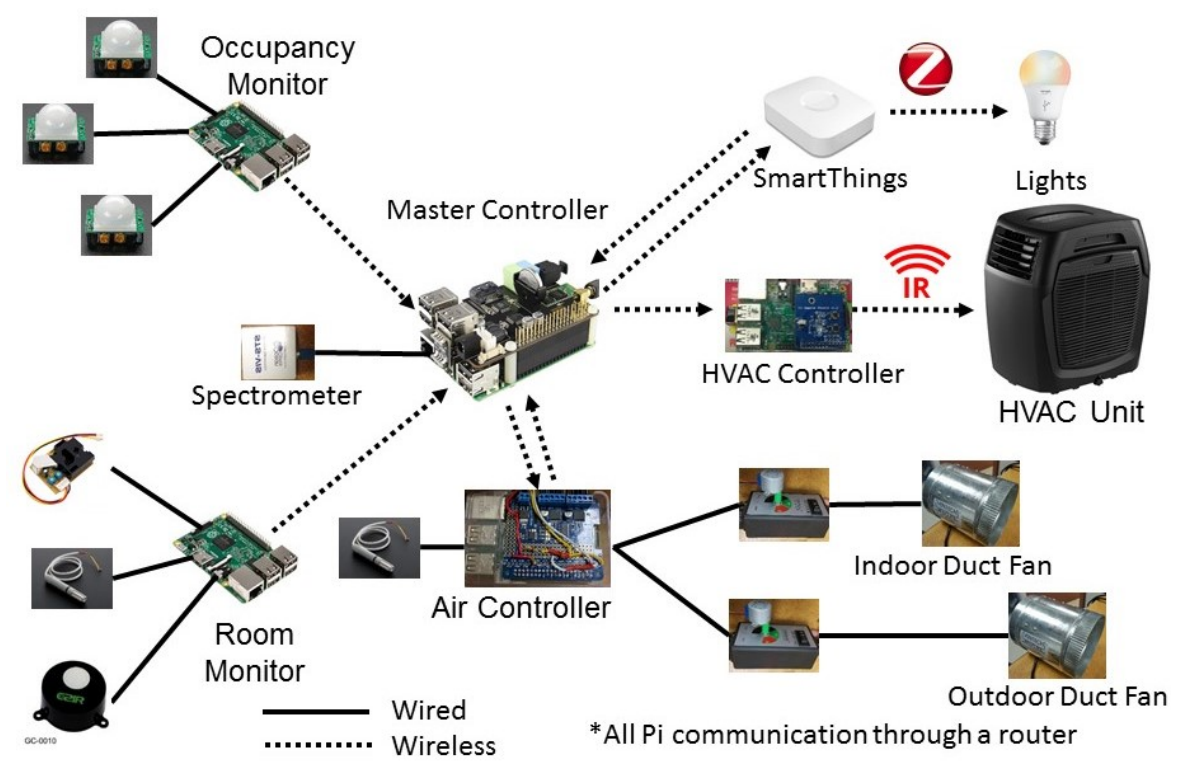

Figure 1. Human Centric Building Control System

U.S. national building codes (NBCs) specifications are based solely on photopic light levels. Table 1 provides a comparison of energy savings for the utilized luminaire between NBC baseline and the lamp producing a numerically equivalent VEL. All measurements were made at $45 \mathrm{~cm}$ from the light source inside the previously described enclosure. The comparison was made at 525 photopic lux and at a constant CCT of $5000 \mathrm{~K}$ which are conditions which are typical in a standard office. The simulated outside light source was set at 4100K (which corresponds to midmorning and midafternoon during the day). For the interior lighting case with no additional outside light provided, a 33\% energy savings was achieved by dimming the lamp until it provided 525 VEL lux. When the simulated exterior lighting was taken into account the VEL case provided a $74 \%$ energy savings over the NBC base case. For comparison, a commercially available system that includes daylighting would achieve a $45 \%$ energy savings in the tested configuration.

Table 1. Demostration of Lighting System Energy Savings

\begin{tabular}{|c|c|c|c|}
\hline & NBC (Watts) & VEL (Watts) & \% Savings \\
\hline No Exterior Light & 6.9 & 4.6 & $33 \%$ \\
\hline With Exterior Light & 3.8 & 1 & $74 \%$ \\
\hline \% Savings & $45 \%$ & $78 \%$ & \\
\hline
\end{tabular}

\section{CONCLUSION}

Office environments with ineffective lighting control systems can produce visually over bright areas. HVAC systems which are ineffective can pose problems for worker productivity and health and increase building operation costs. Controlling the building environment based on total occupancy, visually effective light, and indoor air quality could produce a highly efficient building that also provides physiological and psychological benefits to workers. The HVAC control system developed assures that only the minimum required ventilation rates based on a personal heating load are provided with additional energy savings from the incorporated variable speed controllers. By providing light levels that take into account the contribution rods have on visual perception, the control system can adjust both the spectrum and dimness to reduce power consumption well below current requirements. The utilization of microcomputers and standard sensors resulted in the equipment cost of the developed control system to 
approximately $\$ 4.00$ per $\mathrm{ft}^{2}$. For commercial applications, the developed system cost could be reduced to approximately $\$ 2.50$ per $\mathrm{ft}^{2}$ with the incorporation of an additional equipment.

Further energy consumption optimization could take place with additional characterization of the luminaire for various CCT values and distances between light source and the work plane. In addition, incorporation of an infrared camera to determine the occupancy and real time heat loads from both workers and auxiliary heat loads (e.g. computers) would provide the ability to further trim HVAC energy consumption. The incorporation of wearable sensors to monitor heart rate among other factors could also be included in the system to determine employee alertness and overall health. This data could be used to adjust environmental parameters such as increasing the CCT level and lower the temperature to wake up a worker.

\section{REFERENCES}

[1] G. Rawal, "Costs, Savings, and ROI for Smart Building Implementation”. Available: http://blogs.intel.com/iot/2016/06/20/costs-savingsroi-smart-building-implementation/ 2016.

[2] PR Mills, SC Tomkins, LJ Schlangen, "The effect of high correlated colour temperature office lighting on employee wellbeing and work performance", Journal of Circadian Rhythms, 5:2, 2007.

[3] S. Tom, "Managing Energy and Comfort", ASHRAE Journal, June, pp.18-26. 2008.

[4] U. Satish, M.J. Mendell, K. Shekhar, T. Hotchi, D. Sullivan, S. Streufert, W.J. Fisk, "Is Co2 an indoor pollutant? Direct effects of low-tomoderate CO2 concentrations on human decition-making performace." Enivonmnetal Health Prospect, 120(12) pp, 1671-1677. 2012.

[5] V. Loftness, V. Hartkopf, B. Gurtekin, "Linking Energy to Health and Productivity in the Built Enviornment: Evalutaing the Cost-Benefits of hhigh Performance Building and Community Design for Sustianbility, Health and Productivity.” USGBC Green Build Conference, 2003.

[6] S. Berman, "The coming Revolution in Lighting Practice" Available: https://www.researchgate.net/publication/229018510_The_Coming_Revolution_in_Lighting_Practice 2000.

[7] US Environmental Protection Agency, "Integrated Review Plan for the National Ambient Air Quality Standards for Particulate Matter" Available: $\mathrm{https://www3.epa.gov/ttn/naaqs/standards/pm/data/201612-final-integrated-review-plan.pdf} 2016$.

[8] American Industrial Hygiene Assocaition, "Indoor Environmental Quality / Indoor Air Quality" Available: https://www.aiha.org/publications-and-resources/TopicsofInterest/Topics/Pages/Indoor-Air-Quality.aspx 2017.

[9] American Society of Heating, Refrigerating and Air-Conditioning Engineers, "Indoor Air Quality Guide: Best Practices for Design, Construction and Commissioning" ASHRAE Atlanta, GA, 2009.

[10] American Society of Heating, Refrigerating and Air-Conditioning Engineers, "ANSI/ASHRAE Standard 62.1-2016 Ventilation for Acceptable Indoor Air Quality" Atlanta, GA, ASHRAE/ANSI 2016.

[11] American Society of Heating, Refrigerating and Air-Conditioning Engineers, "Indoor Air Quality Resources." Available: https://www.ashrae.org/resources--publications/bookstore/iaq-resources\#cp2013 2017.

[12] U.S. Green Building Council, "Minimum indoor air quality performance." Available: http://www.usgbc.org/credits/retail-newconstruction/v4-draft/eqp1 20172013.

[13] European Committee for Standardization, "EN 15251:2007 Indoor environmental input parameters for design and assessment of energy performance of buildings addressing indoor air quality, thermal environment, lighting and acoustics." Available: https://standards.cen.eu/dyn/www/f?p=204:110:0\%:.:FSP_PROJECT,FSP_ORG_ID:24552,6138\&cs=1AAF5A672C76C7DC4F78CCAAE6 304DE5D 2007.

[14] European Committee for Standardization, "EN 13779:2007 Ventilation for non-residential buildings - Performance requirements for ventilation and room-conditioning systems." Available:

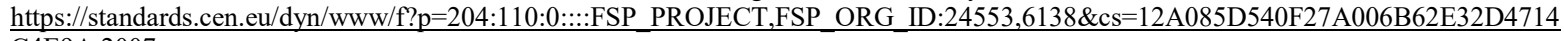
C4E9A 2007.

[15] American Industrial Hygiene Assocaition AIHA. (2017). "Indoor Environmental Quality / Indoor Air Quality " Available: https://www.aiha.org/publications-and-resources/TopicsofInterest/Topics/Pages/Indoor-Air-Quality.aspx 2017.

[16] American Industrial Hygiene Assocaition, "Body of Knowledge-Indoor Air Quality Practitioner." Available: https://www.aiha.org/publications-and-resources/BoKs/IAQ-IEQ/Pages/BoK-IAQ-IEQ.aspx 2015. 